\title{
Water Pollution Detection of Tigris River by Utilizing Thermal Remote Sensing Techniques: North Baghdad-Iraq Region as a Case Study
}

\author{
Mohammed Mejbel Salih 1,2* \\ 1. Geomatics Engineering Division, Civil Engineering Department,University of Technology-Iraq, Baghdad, \\ Iraq. \\ 2. Laser and Optroelectronics Eng.Dept., University of Technology-Iraq, Baghdad, Iraq. \\ *E-mail addresses: 40339@uotechnology.edu.iq
}

Received: 12/09/2020 Accepted: 11/01/2021

DOI: 10.7149/OPA.54.1.51045

\begin{abstract}
:
The land on which we live has a tight environmental system, and the elements of life in it must be preserved, as pollution rates have increased in general, including water pollution, and the environmental problems it causes due to population inflation. The development of modern science and technology can contribute greatly to preserving the components of the environmental balance. It was scientifically found that each body has a radiative value that distinguishes it from other bodies and this is called the thermal fingerprint of objects, and therefore the radiation signature of a body is what distinguishes it from other bodies. Accordingly, from here it is possible to reveal what these objects are without direct contact and know their components from distance. This study addresses the aspect of monitoring the spectral footprint of polluted water, especially the waste of hospitals and factories, which causes great damage to the beaches and must be monitored first-hand through the use of a thermal monitoring device and conducting field examinations directly. The study area was determined near one of the wastes of Baghdad hospitals on one side of the Tigris River and compared with the values of the thermal spectral footprint taken from satellites that carry thermal sensors The results showed the convergence of the measured values with the values taken from the thermal sensors, which confirms the accuracy of the thermal images in the (10-11) beam of the satellite.
\end{abstract}

Key words: Remote sensing; IR thermometer; Landsat-8; Emissivity.

\section{REFERENCES AND LINKS}

[1] J. L. Awange and J. B. Kyalo Kiema, "Fundamentals of remote sensing," Environ. Sci. Eng. (Subseries Environ. Sci., no. 9783642340840, pp. 111-118, 2013.

[2] P. C. Bakker W.H., Janssen L.L.F., Reeves C.V., Gorte B.G.H. and and W. T. Weir M.J.C., Horn J.A., Prakash A., Principles of Remote Sensing. ITC, Enschede, The Netherlands, 2001.

[3] J. R. Jensen, Remote Sensing of the Environment An Earth Resource Perspective, Second Edi. Pearson Education Limited, 2014.

[4] K. K. Lwin, "Fundamentals of Remote Sensing and Its Application in GIS," Div. Spat. Inf. Sci., 2008.

[5] X. Yu, X. Guo, and Z. Wu, "Land surface temperature retrieval from landsat 8 TIRS-comparison between radiative transfer equation-based method, split window algorithm and single channel method," Remote Sens., 2014. 
[] ] A. J. Abdalkadhum, M. M. Salih, and 0. Z. Jasim, "Combination of visible and thermal remotely sensed data for enhancement of Land Cover Classification by using satellite imagery," IOP Conf. Ser. Mater. Sci. Eng., vol. 737, no. 1, 2020.

[7] D. Lu and Q. Weng, "A survey of image classification methods and techniques for improving classification performance," International Journal of Remote Sensing. 2007.

[8] T. M. Lillesand, R. W. Kiefer, and J. W. Chipman, Remote sensing and image interpretation. 2004.

[9] J. A. Sobrino et al., "Land surface emissivity retrieval from different VNIR and TIR sensors," in IEEE Transactions on Geoscience and Remote Sensing, 2008.

[10] Z. Qin, A. Karnieli, and P. Berliner, "A mono-window algorithm for retrieving land surface temperature from Landsat TM data and its application to the Israel-Egypt border region,” Int. J. Remote Sens., 2001.

[11] A. Prakash, “Thermal remote sensing: concepts, issues and applications,” ... Arch. Photogramm. Remote Sens., 2000.

[12] J. P. Walawender, M. Szymanowski, M. J. Hajto, and A. Bokwa, "Land Surface Temperature Patterns in the Urban Agglomeration of Krakow (Poland) Derived from Landsat-7/ETM+ Data,” Pure Appl. Geophys., 2014.

[13] Q. Weng, D. Lu, and J. Schubring, "Estimation of land surface temperature-vegetation abundance relationship for urban heat island studies," Remote Sens. Environ., vol. 89, no. 4, pp. 467-483, 2004.

[14] T. Borowik, N. Pettorelli, L. Sönnichsen, and B. Jędrzejewska, "Normalized difference vegetation index (NDVI) as a predictor of forage availability for ungulates in forest and field habitats," Eur. J. Wildl. Res., 2013.

[15] M. M. Salih, O. Z. Jasim, K. I. Hassoon, and A. J. Abdalkadhum, "Land Surface Temperature Retrieval from LANDSAT-8 Thermal Infrared Sensor Data and Validation with Infrared Thermometer Camera," 2018.

[16] M. Stathopoulou and C. Cartalis, "Daytime urban heat islands from Landsat ETM+ and Corine land cover data: An application to major cities in Greece," Sol. Energy, 2007.

[17] K. Zanter, “Landsat 8 (L8): DATA USERS HANDBOOK,” Mehran Univ. Res. J. Eng. Technol., vol. 37, no. 4, 2018.

\section{Introduction}

Remote sensing techniques or remote sensing is one of the ancient engineering sciences and is included in all other disciplines, and it is simply possible to define remote sensing terms that are vision for objects that cannot be seen [1-2]. The features of remote sensitivity have been concerned with the search and disclosure of the wealth and natural resources on the earth, which have many different activities between the forties and fifties of the last century. The rapid development of computers led to the development of the field of remote sensing techniques, and was a reason for the increase in exploration and detection of ground resources, especially in the field of radar imaging [3], [4]. These technologies of IR require the use of and employing means and supporting equipment to benefit from them in interpreting aerial photos, in addition to that the most important factor is the experience and skill that the data collector must possess while I interpret the images in a detailed. Pioneering step in discovering natural resources began after the launch of many satellites, especially after 1972, which strengthened the capabilities and skills of remote sensing techniques to the best.

The thermal effects on the surface of the globe in addition to exploration have increased in the last ten years effectively and became Satellite images give important and useful details for large areas, as well as they, take pictures periodically and at any time and are multi-spectral $[5,6]$. The presence of digital computer technologies helps to improve the efficiency of the interpretation of thermal satellite images[7], [8]. The presence of thermal sensors in the satellites helps a lot in a new classification channel for everything that is in nature[9].

In terms of the thermal footprint, a substance in the universe that has a thermal spectral signature depends on the relationship that links the temperature of the medium of the body and the temperature of the surroundings[10]. The 10 band and 11 band, that monitor the rise of temperature within wide areas and for the distance between the sensor of this temperature in the satellite and the surface of the earth, may 
give a certain error rate, that is a widely applied procedure with specialists in this field, especially for those working in the field of remote monitoring and control.

To analyse thermal images directly using special programs to then calculate the thermal emissivity at any point and for the results to be acceptable the specialists need to conduct checks at the same time that the satellite takes thermal images presence device RF power meter in the same area for the purpose of knowing the accuracy and efficiency of the thermal sensors of any satellite that gives images of the thermal beams.

On this basis, the researchers designed and built the temperature measurement systems within An infrared beam that gives high accuracy in calculating the temperature difference or contrast between the body and its surroundings. To calculate the emissivity of this object and compare it with the emissivity issued by the thermal sensors, they are converted into thermal images that are entered into the computer to be processed digitally to know the emissive value of the same objects and to know the of the objects accurately. A very advanced temperature measuring device for objects and the surrounding medium with a low error rate. It has been used reading data from device to mathematical relations to determine the accuracy of the convergence of the results. The Tigris River is one of the largest rivers in Iraq and the Arab world, and it divides the city of Baghdad into two halves, namely the Karkh and Rusafa sides. This study use the Remote sensing technique and aim to provide an indication of the thermal behaviour of wastewater and to measure the amount of energy emitted from it. The study site near the Imams Bridge was chosen at coordinates 44.355140 degrees east and 33.372618 degrees north as shown in Fig. (1).

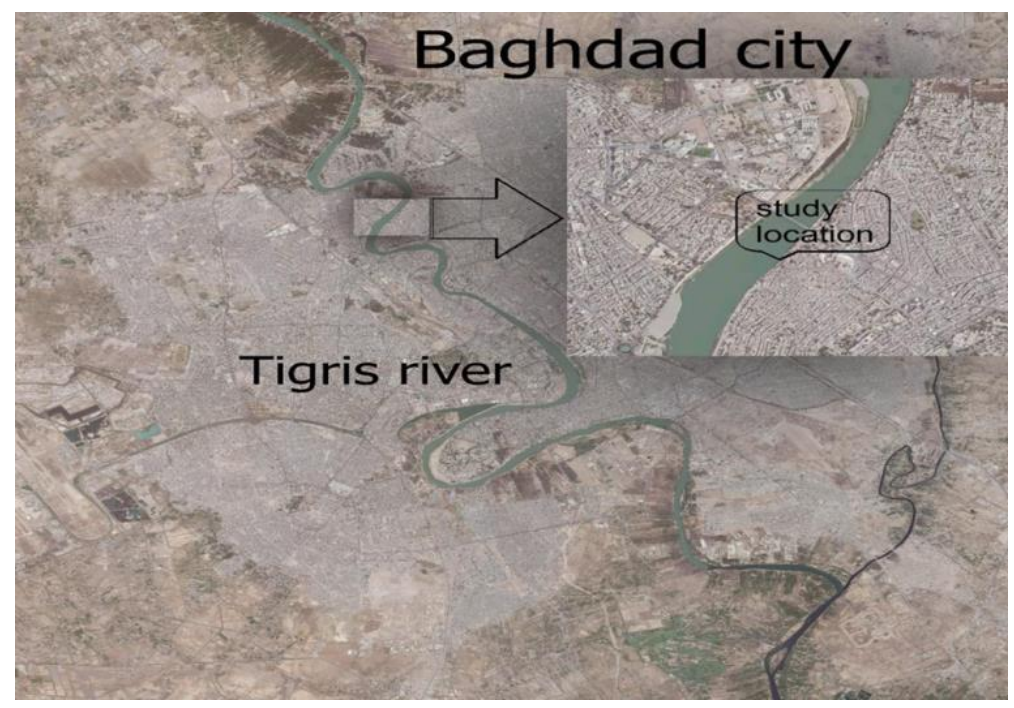

Fig. 1. Baghdad City (Case stady area)

\section{Methodology and Data Set}

The methodology of this study is based on the principles of the infrared remote sensors and its application in water quality studies. Thermal infrared sensors and more recently differentiation of very subtle shifts in spectral responses due to waste using a data-intense high-resolution spectrometer (HRS). Unlike earlier systems, (HRS) has the potential to identify specific materials based on molecular structure, but this is generally difficult due to complex atmospheric interferences. In this paper we will focus on the thermal techniques and applications for measuring and monitoring of wastewater, generally, the work can be divided into two main processes:

\subsection{The process of direct measurement of the surface temperature}

Using the typical temperature measuring devices. Such as the IR-video Thermometer which is a device that can be used to measure the surface temperature of the objects that is improper to be measured by traditional (contact) thermometer, it is capable of non-contact (infrared) temperature measurements with the visual camera at the touch of a button [11]. The process of identifying the surface temperature using satellite image and image processing and manipulating applications. In this paper, it has used data thermal map from a satellite image of the area under study. The data captured on the 9 of December 2016 and 
downloaded from United State Geological Survey (USGS) website. The software used for image processing and interpretation is ArcGIS by Esri.

\subsection{Land Surface temperature}

The purpose from make a comparison to the thermal fingerprint of land surface temperature LST. The heat value was taken through the bands. (10 and 11), the readings were taken from the red band (4) and nearinfrared band (5) represented the Normalized Difference Vegetation Index (NDVI) using the term in any extraction of natural differences to vegetarians and by using the equations of digital conversion from satellites. to the thermal spectral radiance $(L \lambda)$ by using equation [1]:

$$
L_{\lambda}=M_{L} Q+A_{L}
$$

Where $M L=$ multiplicative rescaling factor from data, $Q$ cal= Quantized standard digital number $D N$ value of pixels, $\mathrm{AL}=\mathrm{Band}$ - additive rescaling factor from the dataset.

From equation number (2) we can calculate the brightness temperature (BT) taken from the thermal sensor in satellite [12].

$$
\mathrm{BT}=\frac{K t 2}{\ln \left[\left(\frac{K t_{1}}{L_{\lambda}}\right)+1\right]}-273.15
$$

(Kt1 and Kt2) thermal infrared bands (10 and 11). in Celsius ( $\mathrm{C}^{\circ}$ ) by adding absolute temp. ( 0 to 273 ). NDVI values $(-1$ to +1$)$ ranges from $(-1$ to +1$)$ taken from (RED) band $(0.656-0.674 \mu \mathrm{m})$ and near-IR band $(0.852-0.876 \mu \mathrm{m})$ of images by using equation (3).

$$
\text { N. D. V. I }=\frac{(\text { near IR-RED })}{(\text { nearIR+RED })}
$$

Where NIR is the near-infrared band value of pixel and RED is the band of the same pixel. The NDVI(Normalized difference vegetation index) is essential to calculate proportional plants (PP) and emissivity [13,14].

From NDVI values obtained in the previous step calculate proportional plants (PP). This proportional plant gives the estimation of the area under each land cover type. The plant and bare soil proportions are acquired from the NDVI of the pixels.

Where (s) the soil and (v) vegetation

$$
P P=\left(\frac{N \cdot D V I-N D V I_{s}}{N \cdot D V I_{V}-N D V I_{s}}\right)^{2}
$$

TABLE 1. Data of LANDSAT-8 Satellite image

\begin{tabular}{|c|c|c|c|}
\hline Symbols & Descriptions & $\begin{array}{l}\text { Value of } \\
\text { Band10 }\end{array}$ & $\begin{array}{c}\text { Value } \\
\text { of Band11 }\end{array}$ \\
\hline $\mathrm{K}_{\mathrm{t} 1}$ & Thermal constant & 774.8853 & 480.8883 \\
\hline $\mathrm{K}_{\mathrm{t} 2}$ & A.RADIANC-EMULT & 1321.0789 & 1201.1442 \\
\hline $\mathrm{M}_{\mathrm{L}}$ & D.N value & 0.00033420 & 0.00033420 \\
\hline $\mathrm{Q}_{\mathrm{ual}}$ & RADIANCE-ADD & & \\
\hline $\mathrm{A}_{\mathrm{L}}$ & \multicolumn{2}{|c|}{0.1} & 0.1 \\
\hline
\end{tabular}




\section{Results and dissection}

The objective of this research is to make an indication of the thermal behavior of the wastewater and measuring the amount of energy emitted from it in the principles of the surface temperature, because of the surface temperature is a function of the emissivity of the body. It can make a prediction of the emission for that body represented by an emissivity curve along the day time and by comparing this curve with the emissivity curves of different bodies measured by the thermal remote sensing sensors we can distinguish the wastewater from the other objects in the same single satellite image. This approach provides the following advantages:

a. A very convenient way for non-contact identification of the earth features from the satellite imagery.

b. Facilitate the monitoring process for the earth's features at night and bad weather conditions based on thermal of the water and wastewater using the thermal sensor in the Landsat-8, the thermal sensor in the satellite depends on the rays emitted from the objects and the effect of the clouds at night and day is relatively weak and it is possible for the thermal contrast gas of surfaces and people to use the thermal sensor easily.

c. Facilitate the processes of wastewater management and water quality issues.

\subsection{IR video thermometer}

It is a device that can be used to measure the surface temperature of the objects that is improper to be measured by traditional (contact) thermometer (such as moving object, the surface with electricity current or the objects which are uneasy to be touched). It is capable of non-contact (infrared) temperature measurements with a visual camera at the touch of a button. The built-in laser pointer increases target accuracy while the backlight LCD and handy push-button combined for convenient and ergonomic operation (Fig. 2).

In this process, we simply measure the surface temperature of the area under study which is the wastewater by using a surface temperature measuring device which is called (IR video thermometer). This device allows a remote measuring of the surface temperature and has other capabilities we will talk about it.

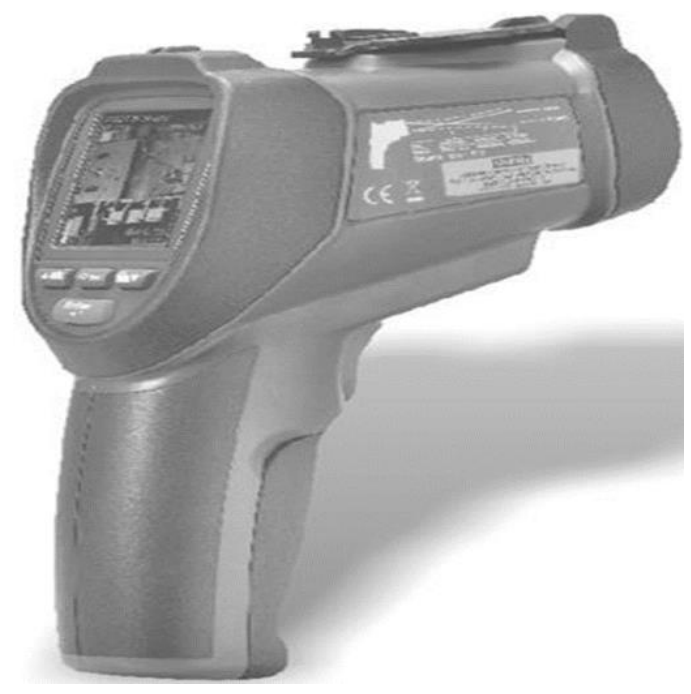

\subsection{Landsat-8}

Fig 2. IR-video thermometer

Using Landsat-8 satellite imagery to gathered the land surface temperature Fig. (3) and Fig. (4) LST for area interested and compared this data with the surface temperature measured directly by using IRthermometer in time of image from 9/12/2019. For instance, it showed good compatibility between the results of the satellite and IR thermometer [15]. Land surface emissivity give us the radiative absorption ability of a surface in the loge wave [9]. LSE is largely dependent on the target surface top layer such as the type of soil, surface roughness, and nature of vegetation cover $[16,17]$. 


$$
\begin{array}{r}
\text { ÓPTICA PURA Y APLICADA } \\
\text { www.sedoptica.es } \\
\varepsilon=\varepsilon_{v \lambda} \cdot P_{V}+\varepsilon_{s \lambda} \cdot\left(1-P_{V}\right)+C_{\lambda}
\end{array}
$$

Where:

$\varepsilon=$ Land Surface Emissivity,

$\varepsilon_{\mathrm{s} \lambda}=$ soil emissivity

$\varepsilon_{\mathrm{v} \lambda}=$ plant emissivity

$\mathrm{PV}=$ proportion of plants

$\mathrm{C}_{\lambda}=$ surface constant $(0.009)$

The NDVI can be used to estimate the emissivity of different land surfaces in the 10-12 $\mu \mathrm{m}$ range (Sobrino et al., 2008).

$$
\varepsilon_{\lambda}=\left\{\begin{array}{cc}
\varepsilon_{S \lambda} & N D I<N D V I_{S} \\
\varepsilon_{S \lambda} P_{V}+\varepsilon_{S \lambda}\left(1-P_{V}\right)+C_{\lambda} N D V I_{S} \leq N D V I \leq N D V I_{V} \\
\varepsilon_{S \lambda}+C_{\lambda} & N D V I>N D V I_{V}
\end{array}\right.
$$

for final step using (B.T) of 2 bands (10,11, and LSE) it is derived from NDVI [16]. using equation (7).

$$
\mathrm{LST}=\frac{B T}{\left[1+\left\{\left(\frac{\lambda B T}{\rho}\right) \ln \varepsilon_{\lambda}\right\}\right]}
$$

BT in Celsius ( $\left(C^{\circ}\right),(\lambda)$ is the wavelength, $(\varepsilon \lambda)$ is the emissivity that computed at eq. (5), $(\rho)$ is $\left(\mathrm{h} x \frac{\mathrm{c}}{\sigma}\right)$ which is $=1.438 \times 10-2 \mathrm{MK},(\sigma=1.38 \times 10-23 \mathrm{~J} / \mathrm{K})$, (h) Plank low $=6.625 \times 10 \mathrm{E}-34$ and $\mathrm{c}=3 \times 108 \mathrm{~m} / \mathrm{s}$.

The thermal data of land surface extracted from (LST-8) for (10\&11) are a camera with the measurement of surface temperature by using the IR-video thermometer camera and the results as shown in Table (2).

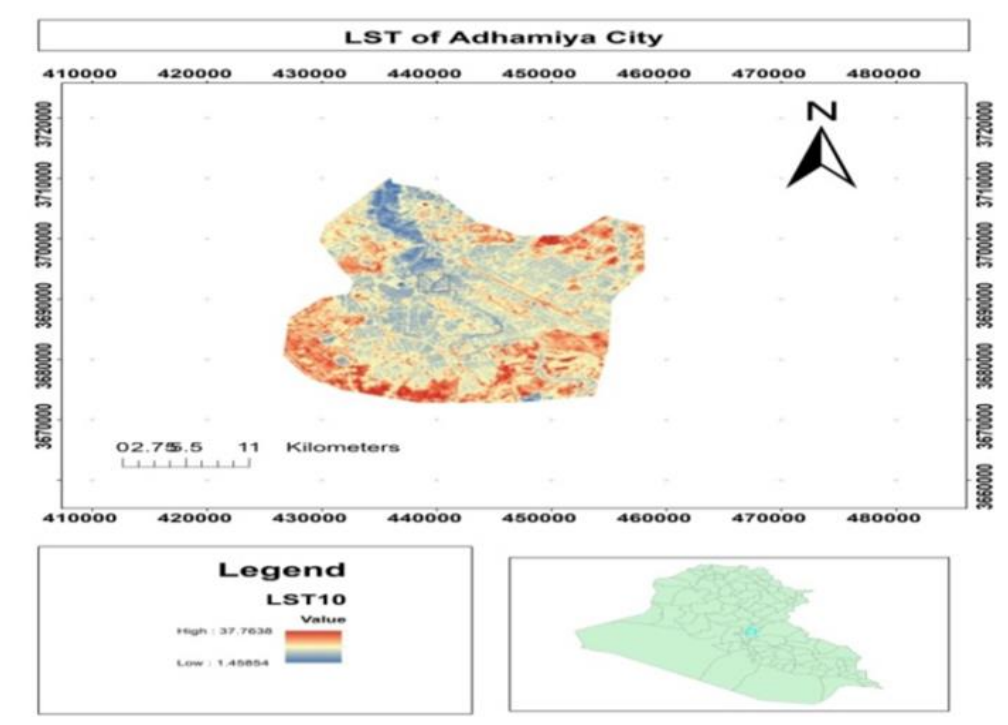

Fig. 3 LST_Band10 of the area interested 


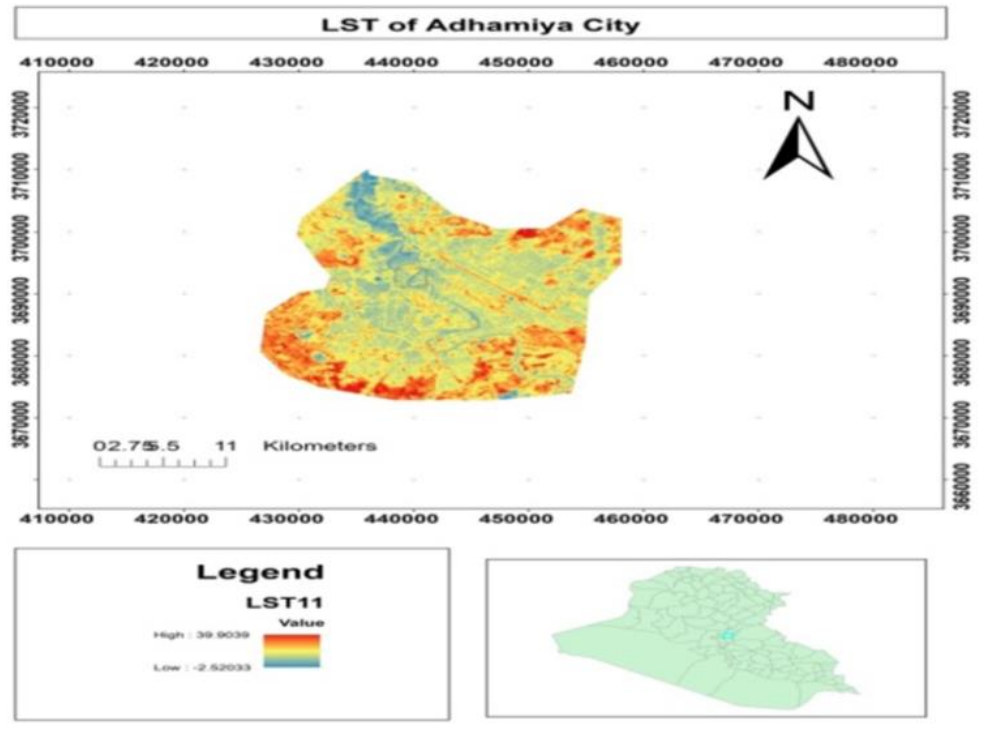

Fig. 4. LST_Band11 of the area interested

The results are shown in Table (2) uncovered that there is a linear intermediate correlation equation of medium strength between the band 10 of satellite image and measurements taken by the IR_thermometer camera as in Fig. (5) where $\mathrm{R}^{2}=0.396$. Given the convergence of the difference between the temperatures, as well as monitoring the passage of the satellite and taking the image simultaneously on this date, in addition to the field measurements. For this reason, the readings were adopted on this date and they represent the thermal spectrum footprint in this month of the surface of polluted water.

TABLE 2. LST Data computed and extracted from Landsat-8 and IR-video thermometer

\begin{tabular}{|c|c|c|c|c|c|c|c|}
\hline $\mathrm{X} \mathrm{m}$. & Y m. & $\begin{array}{c}\text { LST from } \\
\text { Band } 10\left(\mathrm{C}^{\circ}\right)\end{array}$ & $\begin{array}{c}\text { LST from } \\
\text { Band } 11\left(\mathrm{C}^{\circ}\right)\end{array}$ & Av. & $\begin{array}{c}\text { Surface temperature } \\
\text { by using IR-video } \\
\left.\text { thermometer( } \mathrm{C}^{\circ}\right)\end{array}$ & $\begin{array}{c}\text { LST10 - } \\
\text { IR } \\
\left(\mathrm{C}^{\circ}\right) \\
\end{array}$ & $\begin{array}{l}\text { LST1 } \\
1-\text { IR } \\
\left(\mathrm{C}^{\circ}\right) \\
\end{array}$ \\
\hline 440416 & 3693536 & 11.41 & 10.92 & 11.165 & 12.1 & -0.69 & -1.18 \\
\hline 440342 & 363367 & 10.84 & 10.48 & 10.66 & 11.8 & -0.96 & -1.32 \\
\hline 440236 & 3693250 & 11.13 & 10.63 & 10.88 & 12.2 & -1.07 & -1.57 \\
\hline 440151 & 3693166 & 11.41 & 10.89 & 11.15 & 12.4 & -0.99 & -1.51 \\
\hline 439966 & 3692843 & 10.31 & 10.26 & 10.285 & 11.1 & -0.79 & -0.84 \\
\hline 439850 & 3692843 & 11.28 & 10.76 & 11.02 & 12.2 & -0.92 & -1.44 \\
\hline 439844 & 3692742 & 10.96 & 10.49 & 10.725 & 11.3 & -0.34 & -0.81 \\
\hline 439733 & 3692615 & 10.94 & 10.48 & 10.71 & 11.6 & -0.66 & -1.12 \\
\hline 439696 & 3692499 & 10.89 & 10.39 & 10.64 & 12.1 & -1.21 & -1.71 \\
\hline 439564 & 3692340 & 11.21 & 10.33 & 10.77 & 12.3 & -1.09 & -1.97 \\
\hline 439495 & 3692086 & 11.12 & 10.5 & 10.81 & 12.4 & -1.28 & -1.9 \\
\hline 439739 & 3691271 & 10.7 & 10.13 & 10.415 & 12.4 & -1.7 & -2.27 \\
\hline 440056 & 3691261 & 11.3 & 10.87 & 11.085 & 12.5 & -1.2 & -1.63 \\
\hline 440405 & 3691388 & 10.85 & 10.94 & 10.895 & 12.2 & -1.35 & -1.26 \\
\hline
\end{tabular}

This means that there is an average correlation coefficient of forecasts to measure the surface temperature and the reason behind this correlation is because field measurements were collected within an hour during day time, while the image was captured in an estimated time in seconds or less. Therefore, the differences of measurements are clear using an IR-video thermometer. Also, reason is the relative temperature difference. In general, there are many factors that affect the temperature emitted from objects that is the thermal leakage due to winds and shade areas due to intermittent clouds, which leads to divergence in the results relatively and they have little effect on affinity values.

So, the results revealed a weak correlation coefficient it is about $\mathrm{R}^{2}=0.127$ between the thermal temperature at band 11 of satellite image and the measurements taken by the IR-video thermometer camera as in Fig. 
(6), the weak measurements due to the reasons mentioned above, as well as the fact that the band-11was affected by the lighting of stars.

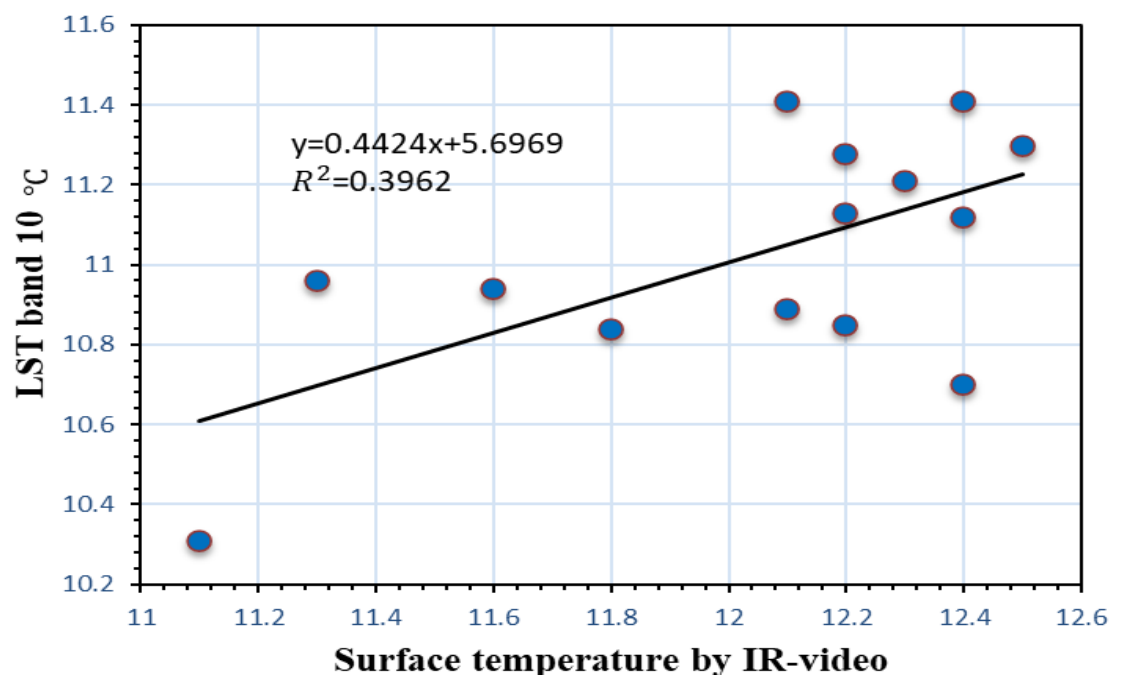

Fig. 5. The correlation coefficient between the temperature of Landsat- 8 band 10 satellite image and IR_camera

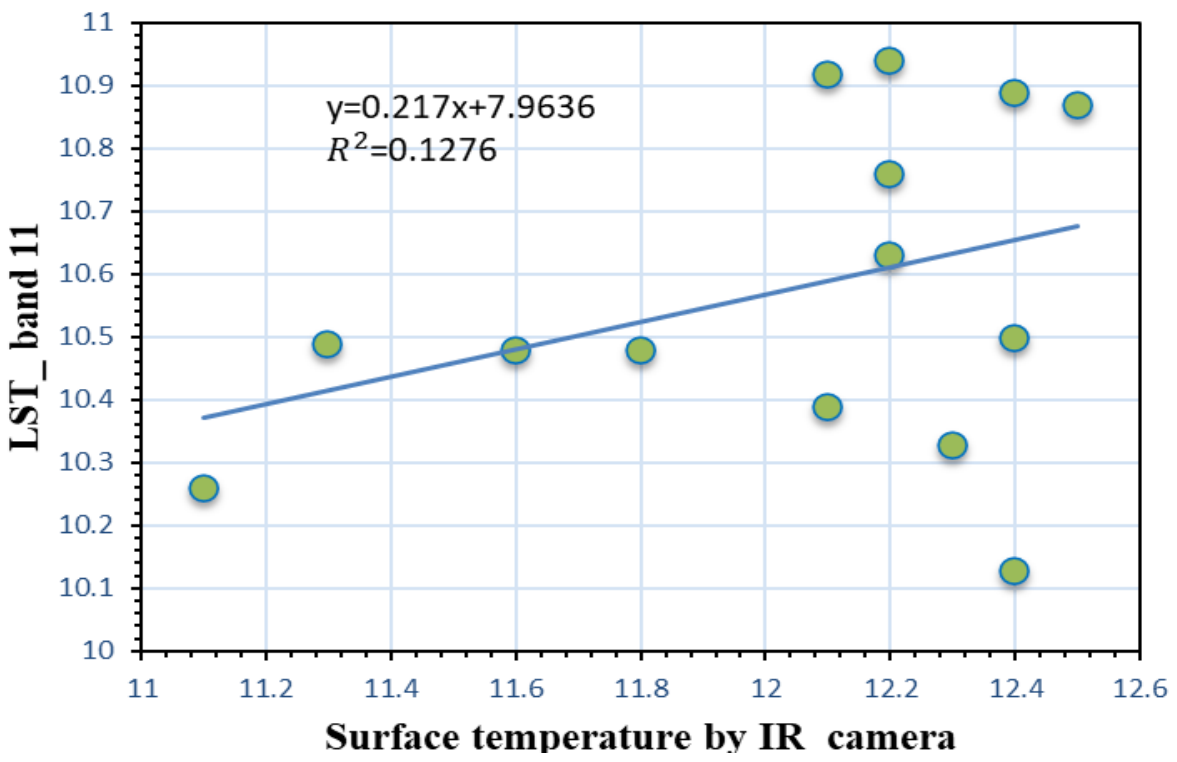

Fig. 6. The correlation coefficient between the temperature of Landsat- 8 band 11 satellite image the IR_camera

From Fig. (7), which represents the readings of the thermal sensor in the Landsat-8, as indicator gives the exact difference to evaluate whether the other bands, for example, that band- 10 is much better than Band -11 . 


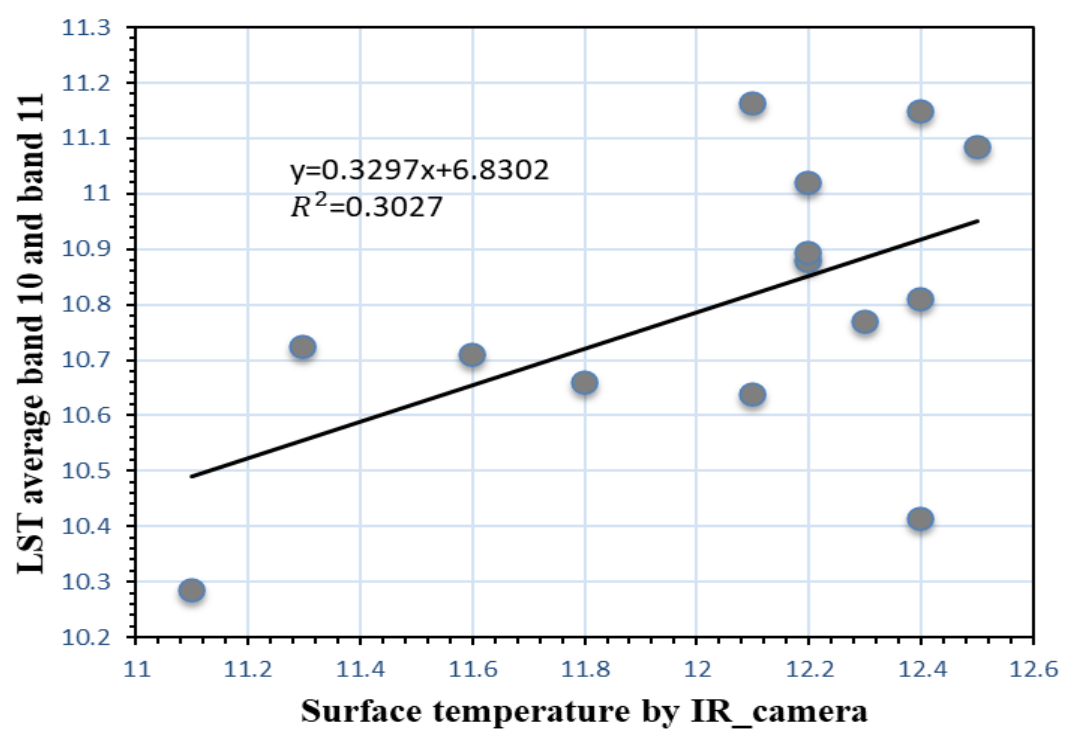

Fig. 7. The correlation coefficient between surface temperature from Landsat- 8 (average bands 10 and 11) satellite image and IR_camera

\section{Conclusion}

The purpose of this research is to make an indication of the thermal behavior of the wastewater and measuring the amount of energy emitted from it in the principles of the surface temperature, because of the surface temperature is a function of the emissivity of the body, we can make an emission signature for that body represented by an emissivity curve along the day time and by comparing this curves with the emissivity curves of different bodies measured by the thermal remote sensing sensors we can distinguish the wastewater from the other objects in the same single image, this process can be done by measuring the surface temperature directly from the area of study by using the thermometer, this direct measurement is done for both wastewater and the ambient temperature to calculate the emissivity of the marital. The emissivity of the wastewater also calculated from a satellite image taken for the same area and nearly at the same time and gives approximately the same surface temperature which means almost equal emissivity, process Autocorrelation Why is it originally the result of taking readings when combined with field tests of polluted water in the Tigris River to ensure the accuracy of the thermal satellite images and that the comparison process is based on the results of equations implicit in the Excel program and The equations (5),(6) show the calculation of the heat emitted from the bodies, especially from the surfaces of the polluted water, to determine the thermal spectral signature of the general pollution in the river water that Satellite images taken from the thermal sensor.

\section{Acknowledgements}

The authors would like to thank the University of Technology-Iraq for their support in this work.

\section{List of Abbreviation}

\begin{tabular}{|l|c|}
\hline HRS & High-resolution spectrometer \\
\hline USGS & United State Geological Survey \\
\hline LST & land surface temperature \\
\hline NDVI & Normalized Difference Vegetation Index \\
\hline BT & Brightness temperature \\
\hline DN & Value of pixels \\
\hline L $\lambda$ & Thermal spectral radiance \\
\hline$\varepsilon$, LSE & Land Surface Emissivity \\
\hline$\varepsilon_{s \lambda}$ & Soil emissivity \\
\hline$\varepsilon_{v \lambda}$ & Plant emissivity \\
\hline PV & Proportion of plants \\
\hline$C_{\lambda}$ & Surface constant (0.009) \\
\hline
\end{tabular}

